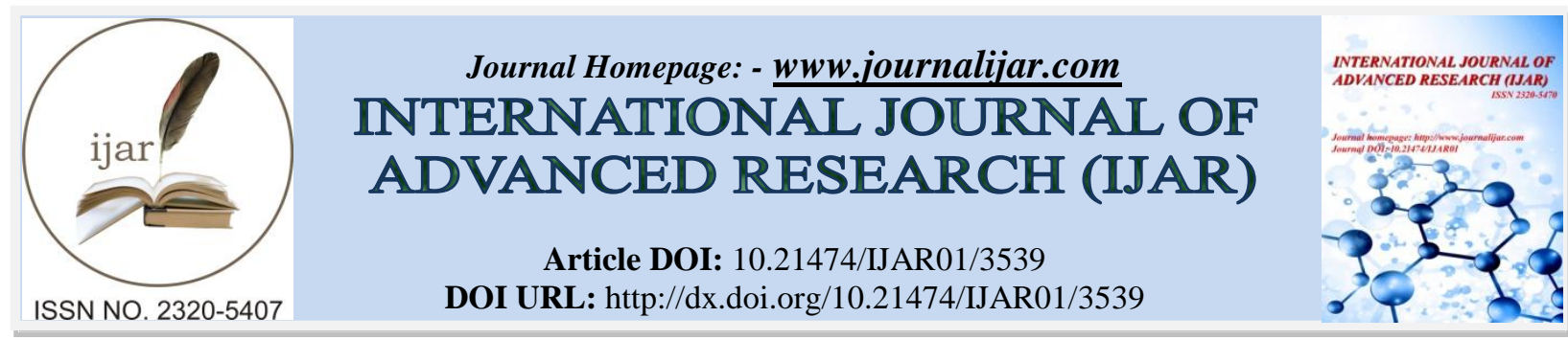

RESEARCH ARTICLE

\title{
OMEGA-3 FATTY ACID IMPROVE NON ALCOHOLIC FATTY LIVER INDUCED BY HIGH FAT DIET IN RATS.
}

Reem M. Hashem ${ }^{1}$, Laila A. Rashed ${ }^{2}$, Ghada M. Safwat ${ }^{3}$ and Ibrahim T. Ibrahim ${ }^{1}$.

1. Department of Biochemistry, Faculty of Pharmacy, Beni-Suef University, Beni Suef, Egypt.

2. Department of Biochemistry, Faculty of Medicine, Cairo University, Cairo, Egypt.

3. Department of Biochemistry, Faculty of Veterinary medicine, Beni-Suef University, Beni Suef, Egypt.

\section{Manuscript Info}

Manuscript History

Received: 16 January 2017

Final Accepted: 14 February 2017

Published: March 2017

Key words:-

NAFLD, Omega-3 fatty acid, glucose, triglycerides, NEFA

\begin{abstract}
Dietary composition can influence the development of Nonalcoholic fatty liver disease (NAFLD). The amount and type of dietary fat may directly affect liver fat content, with high-fat diets being potentially harmful. A higher dietary fat intake is associated with liver inflammation and NAFLD. An Omega-3 fatty acid used in this study as a treatment to modulate these metabolic disturbances. This study was designed to elucidate the effect of Omega-3 fatty acid on high fat diet induced NAFLD in albino Wister rats. NAFLD induced by allowing rats to feed on the high fat diet for 20 weeks. Omega-3 fatty acid was used in doses of $0.8 \mathrm{mg} / \mathrm{Kg}$ body weight. Plasma glucose , triglycerides and non-esterified fatty acids (NEFA) were measured by enzymatic method. It can be concluded that administration of high fat diet caused NAFLD in rats via abnormal or extensive fat accumulation when energy intake exceed energy expenditure. Omega-3 fatty acid succeeded in ameliorating the deleterious effects of high fat diet induced NAFLD.
\end{abstract}

Copy Right, IJAR, 2017,. All rights reserved.

\section{Introduction:-}

Nonalcoholic fatty liver disease (NAFLD) is rapidly becoming a serious global health problem, as its prevalence has risen rapidly in concert with the prevalence of obesity and type 2 diabetes. Estimates of the prevalence of NAFLD range widely from 11 to $46 \%$. It can progress to advanced liver disease in approximately 15-20\% of patients. Clinical characteristics associated with NAFLD include obesity, hyperlipidemia, diabetes mellitus, and hypertension, all of which have been associated with underlying insulin resistance. [1,2]

Aside from weight gain and obesity, dietary composition can influence the development of NAFLD. The amount and type of dietary fat may directly affect liver fat content, with high-fat diets being potentially harmful[3]. A higher dietary fat intake is associated with liver inflammation and NAFLD[4,5]

While no human studies have linked NAFLD and diets high in saturated fat, evidence from experimental animal studies demonstrates that high dietary saturated fatty acid consumption worsens insulin resistance, NAFLD, and cardiovascular disease in rodents[6].

Corresponding Author:- Ibrahim T. Ibrahim.

Address:- Department of Biochemistry, Faculty of Pharmacy, Beni-Suef University, Beni Suef, Egypt. 
Omega-3 fatty acids have recently been proposed as a potential treatment for NAFLD. These fatty acids have proven benefit in lowering serum blood glucose, serum triglycerides nonesterified free fatty acids(NEFFA) and in the treatment of cardiovascular disease. Interest in their potential in the treatment of cancer, mood disorders and cognitive disorders has also emerged.[7]

n-3 polyunsaturated fatty acids (PUFAs), such as docosahexaenoic acid (DHA), have been found to decrease liver fat content in children with NAFLD and possess anti-inflammatory effects. Moreover, recent studies indicated the benefits of n-3 PUFAs in lipid metabolism modification, dyslipidemia improvement, and hepatic inflammation mitigation, which may contribute to the amelioration of NAFLD.[8,9]

The aim of this study is to investigate the biochemical effect of omega-3 fatty acid involved in treatment of NAFLD induced by high fat diet in rats.

\section{Materials and Methods:- \\ Materials:-}

Omega-3 fatty acid was purchased from Sedico Co. (Giza, Egypt). Glucose kits were purchased from Spinreact Co. (Santa Coloma, Spain), triglyceride kits obtained from Spinreact Co. (Santa Coloma, Spain).

\section{Animals:-}

The present study was carried out on Thirty male Wistar rats of $96 \pm 10 \mathrm{gm}$ as body weight range. They were obtained from animal house of research institute of ophthalmology (Giza, Egypt). They were housed in groups of five rats per cage under controlled environmental condition of air and temperature with a $12 \mathrm{~h}$ light- dark cycle. Animal were allowed the diet and water in a free manner. Body weight of rats was recorded every two weeks.

\section{Diet:-}

In this experiment there were the standard normal rat chow diet and the high fat diet for induction of obesity in rats. The standard normal chow diet (ATMID Company, Egypt) consists of soybean, Corn, soybean oil, calcium carbonate, dicalcium phosphate, sodium chloride, lecithin, methionine, and vitamin/mineral mixture.

The high fat diet consists of $25 \%$ fat "beef tallow" $+10 \%$ sucrose $+20 \%$ corn starch $+45 \%$ normal chow with $30 \%$ sucrose in drinking water according to[10] with modification.

\section{Experimental design and animal grouping:-}

The rats were divided randomly into three groups of ten rats each and treated as follows:

Group I:- (normal control): rats were fed on a normal chow diet for 20 weeks and administered water as a vehicle via oral gavage.

Group II:- (High fat diet group): rats were fed on the high fat diet for 20 weeks and administered water as a vehicle via oral gavage.

Group III:- (omega-3-fatty acid treated group): rats were fed on the high fat diet for 20 weeks and administered fish oil daily for the last two consecutive weeks via oral gavage in a dose accounting for $(0.8 \mathrm{gm} / \mathrm{kg}$ body weight) of Omega-3fatty acid[11, 12] The rats were weighed then sacrificed at the end of the experiment after about 18 hours fasting to minimize variation in lipid pattern during two successive days after blood samples have been collected from retro - orbital veins into tubes containing EDTA and centrifuged at 1500 RPM for 30 minutes. An aliquot of the separated plasma was used for immediate estimation of fasting blood glucose. Second aliquot was kept on $-20^{\circ} \mathrm{C}$ for triglyceride and NEFA analysis. All animal procedures were performed upon approval from the Ethics Committee of Beni-Suef University and in accordance with the recommendations of the proper care and use of lab animals.

\section{Biochemical Analysis:-}

Fasting blood glucose was carried out by enzymatic method (Spinreact, Santa Coloma,Spain). The triglyceride level was determined according to the enzymatic method (Spinreact, Santa Coloma, Spain). And NEFA levels were determined according to the (Duncombe) method described by (Duncombe 1964[13]. 


\section{Statistical Analysis:-}

Data were presented as means \pm SEM values. The results were analyzed statistically by one-way analysis of variance (ANOVA) with subsequent multiple comparisons using Tukey multiple comparison Post-Hoc test. The pvalues less than 0.05 were considered significant. Correlations between variables were assessed by Pearson's correlation test. All calculations were made using the computer program SPSS 16.0 (SPSS, Chicago, III, USA). The data were graphed using GraphPad Prism 6 (GraphPad Software, Inc., USA).

\section{Results:-}

Metabolic Markers:-

Fasting plasma glucose level:-

The fasting plasma glucose level was significantly increased in high fat diet treated group when compared to that of the normal control group as shown in table (1). Treatment with omega-3 fatty acid significantly reduced fasting plasma glucose elevation as shown in table (1) and fig. (1).

Table 1:- fasting plasma glucose, triglyceride and non-esteriefied fatty acid levels in the rat of various studied groups.

\begin{tabular}{|l|l|l|l|}
\hline & Normal control & High fat diet group & $\begin{array}{l}\text { Omega-3 fatty acid treated } \\
\text { group }\end{array}$ \\
\hline Fasting plasma glucose level & $93.01 \pm 3.89$ & $135.26 \pm 2.54^{\#}$ & $102.69 \pm 4.12^{*}$ \\
\hline Plasma triglyceride & $84.01 \pm 15.80$ & $145.26 \pm 18.78^{\#}$ & $85.55 \pm 7.12^{*}$ \\
\hline Plasma NEFA & $52.07 \pm 5.89$ & $95.26 \pm 4.54^{\#}$ & $62.69 \pm 4.12^{*}$ \\
\hline $\begin{array}{l}\text { Data are means } \pm \text { SEM. } \\
\text { Data are expressed as mg/dl. }\end{array}$ & \\
$\quad \begin{array}{l}* p<0.05 \text { compared with the normal control group. } \\
* p 0.05 \text { compared with the high fat fed group. }\end{array}$ \\
\hline
\end{tabular}




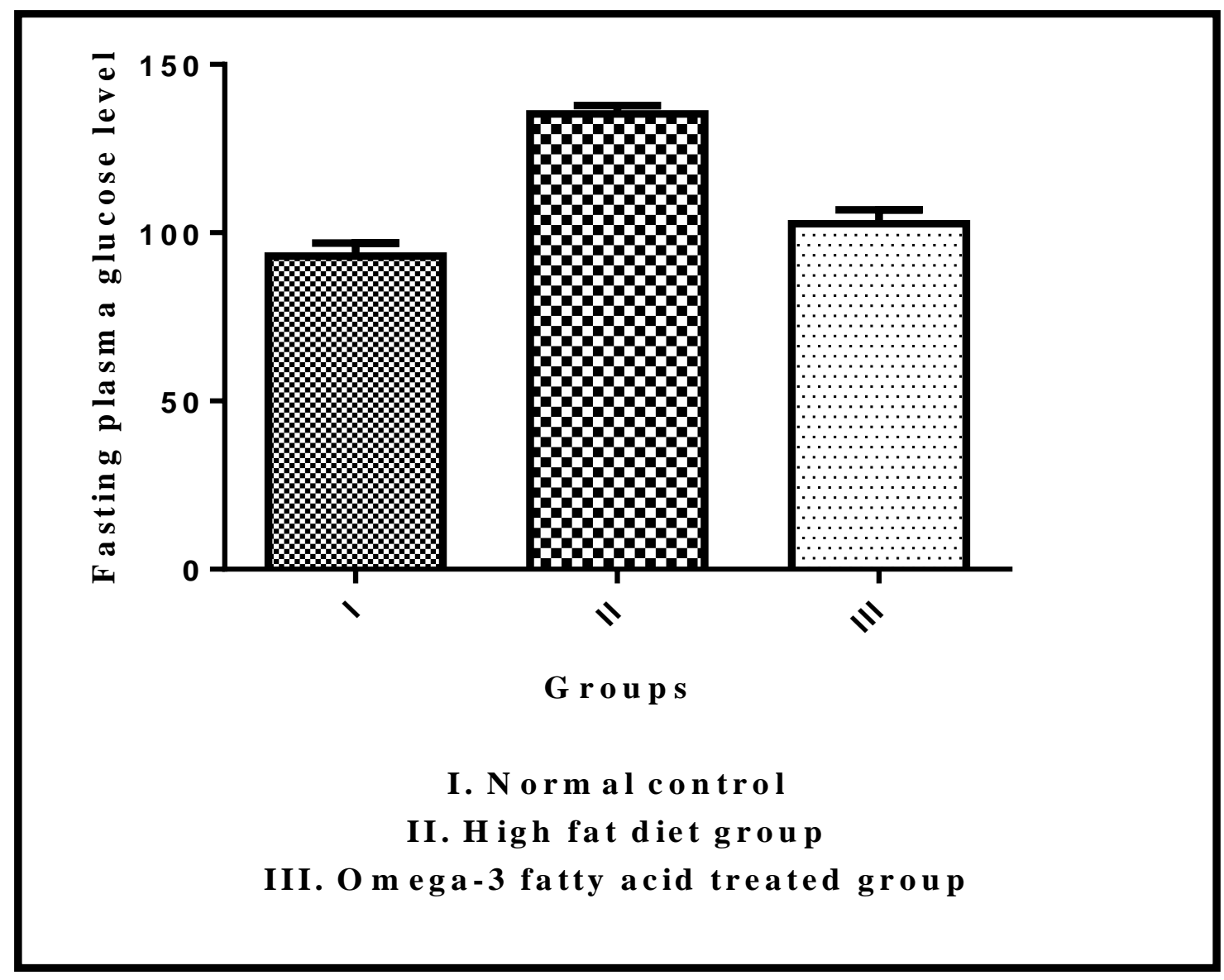

Figure 1:- Changes in fasting plasma glucose level in different groups.

Plasma triglycerides level:-

The plasma triglycerides level was significantly increased in high fat diet treated group when compared to that of the normal control group as shown in table (1). Treatment with omega-3 fatty acid succeeded in significantly reducing plasma triglycerides level as shown in table (1) and fig. (2).

\section{Plasma non- esterified fatty acid level:-}

The plasma non-esterified fatty acid level was significantly increased in high fat diet treated group when compared to that of the normal control group as shown in table (1). Treatment with omega-3 fatty acid succeeded in significantly reducing plasma non- esterified fatty acid level as shown in table (1) and fig. (3). 


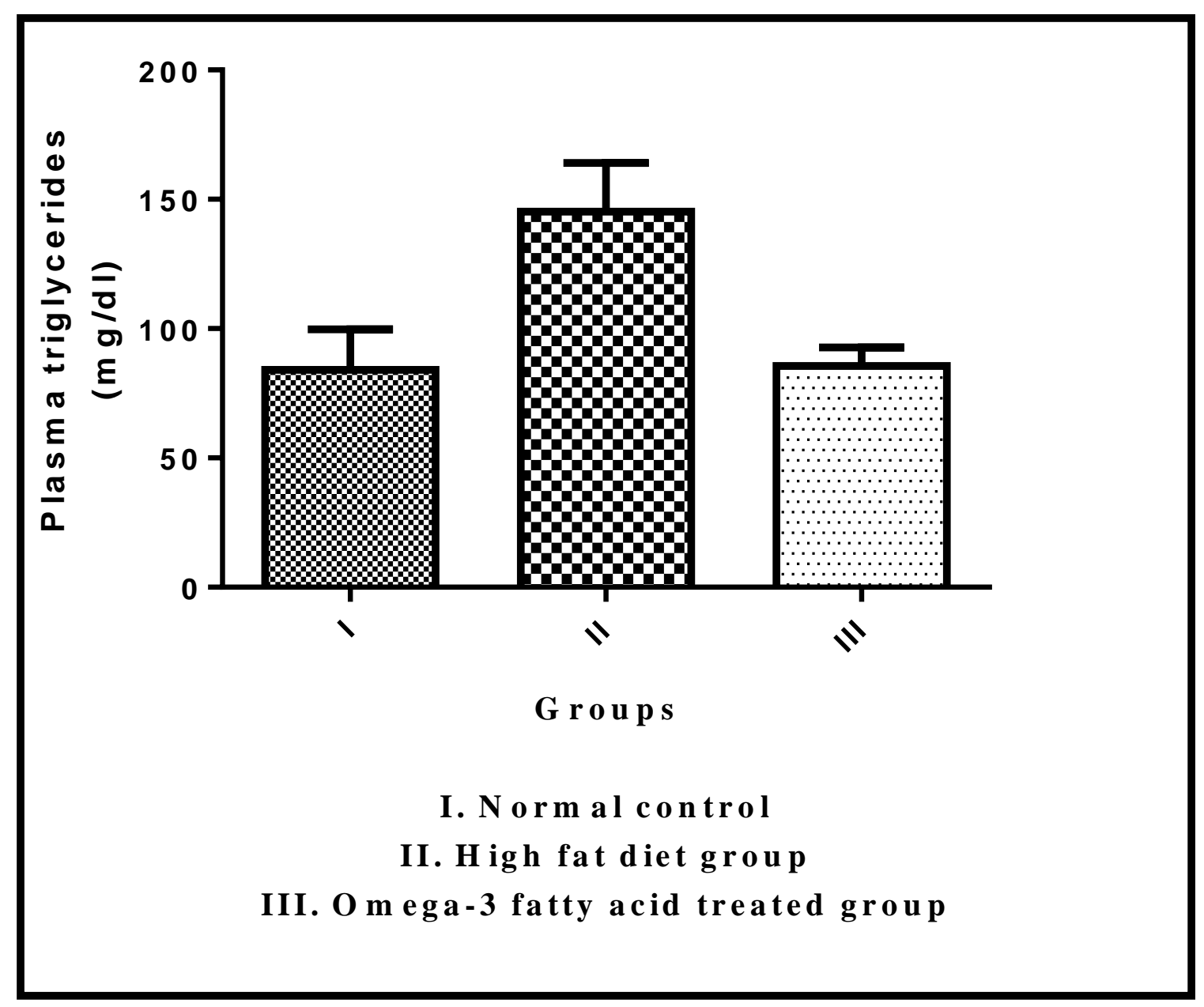

Figure 2:- Change in plasma triglycerides in different groups. 


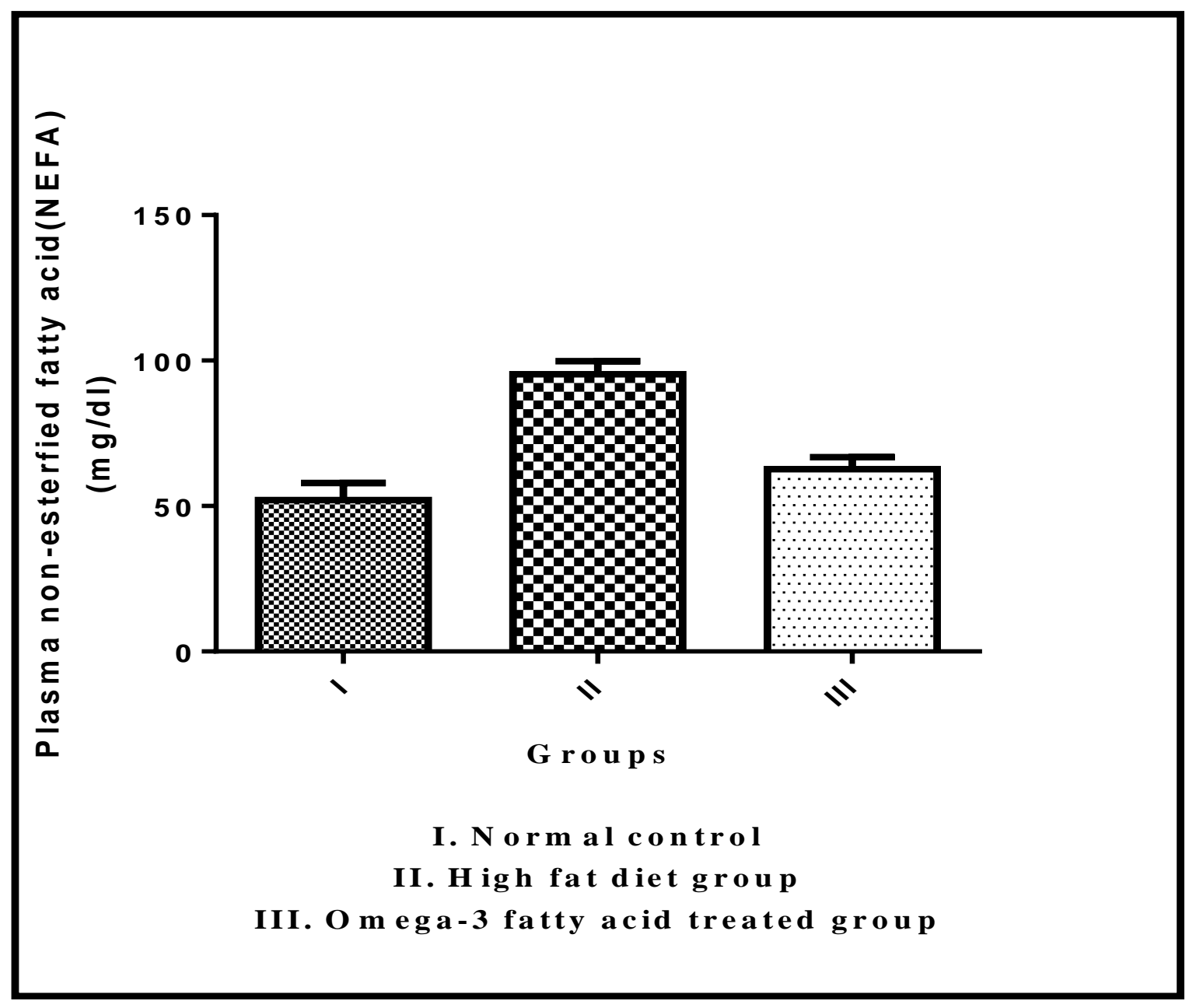

Figure 3:- change in plasma non -esterified fatty acid in different groups.

\section{Discussion:-}

NAFLD can be considered the hepatic manifestation of the metabolic syndrome. Component of metabolic syndrome include insulin resistance, dyslipidemia, type 2 diabetes, hypertension and hypertriglyceridemia[13,14]. When the liver gets fatty because of NAFLD, the ability of insulin to inhibit hepatic glucose production is impaired. This hepatic insulin resistance leads to a slight increase in plasma glucose concentrations and stimulation of insulin secretion. The present study demonstrated that the level of fasting plasma glucose the high fat diet treated group was significantly increased compared to a normal control group. These data were in harmony with the previous study [15]which reported that a significance increase in fasting plasma glucose level with a defect in insulin signaling occurred in adipose tissue after HFD intake in rats compared with of control rats.

The present study found that treatment with omega-3 fatty acid, there was a significant decrease in the raised level of plasma glucose compared to HFD treated group. This in agreement with the previous studies [16,17] ,which reported that omega 3 fatty acid enhanced glucose uptake and improve insulin resistance in HFD-induced NAFLD in rats as omega -3 fatty acid may improve insulin signal transduction in adipocytes, affecting in turn , insulinstimulated glucose transporter (GLUT4) in both skeletal muscule and adipose tissue accompanied with lower glycemia and insulinemia [18]. Moreover, omega-3 fatty acid could regulate both the activity and expression of the liver glucose-6-phosphatase, wich could explain the protective effect with respect to the excessive hepatic glucose output induced by a high fat diet [19].

Hypertriglyceridemia and high levels of non-esterified fatty acids (NEFA) in the MetS promote abnormal accumulation of lipids within the liver, in a form of steatosis or non-alcoholic fatty liver disease (NAFLD) 
(12).Thus, NAFLD affects up to $90 \%$ of obese people and nearly $70 \%$ of the overweight subjects, and it is thought to be the hepatic event in the MetS. In addition, NAFLD is commonly associated with MetS risk factors such as obesity, IR, hypertension and dyslipidemia[20]. Data presented in this study indicated that HFD intake resulted in a significant increase in the level of triglycerides and NEFA in rats compared to those of normal control. The observation of the current study was in accordance with those of the previous studies [21,22] which reported that there is an elevation of both triglycerides and NEFA in NAFLD in rats. The mechanisms governing the accumulation of TGs in hepatic cells include changes in hepatocellular metabolism, [23,24] propagating an imbalance between uptake and de novo fatty acid synthesis, VLDL formation and subsequent export, and oxidation capacity [25].The present study also revealed that treatment with omega-3 fatty acid showed significant reduction in the level of triglycerides and NEFA in plasma compared to those of HFD treated group. This is in agreement with the previous [26,27] which supports the triglyceride-lowering properties of omega-3 fatty acid and decreased NEFA level in plasma. This could be explained by changes in transcription of several nuclear receptors are reported to mediate the TG-reducing effects of omega-3 fatty acid: sterol regulatory element binding proteins (SREBP), liver X receptor-alpha $(\mathrm{LXR} \alpha)$, retinoid $\mathrm{X}$ receptor alpha $(\mathrm{RXR} \alpha)$, farnesoid $\mathrm{X}$ receptor $(\mathrm{FXR})$, and peroxisome proliferator-activated receptors (PPARs), and each play prominent roles in controlling lipid metabolism. Also the reduction of NEFA by omega-3 fatty acid due to suppressing in gene expression involved in new fatty acid synthesis and by inducing fatty acid oxidation in different tissues, such as, liver, skeletal muscle and white adipose tissue [28,29]

\section{References:-}

1. SCHENKER, M. Nonalcoholic steatohepatitis: What we know in the new millennium. The American Journal of Gastroenterology, (2002) 97(11), 2714-2724.

2. McAvoy, N. C.; Ferguson, J. W.; Campbell, I. W. and Hayes, P. C. Non-Alcoholic fatty liver disease: natural history, pathogenesis and treatment. Br J Diabetes Vasc Dis, 2006; 6 (6) , 251-260.

3. Donnelly KL, Smith CI, Schwarzenberg SJ, Jessurun J, Boldt MD, Parks EJ. Sources of fatty acids stored in liver and secreted via lipoproteins in patients with nonalcoholic fatty liver disease. J Clin Invest. 2005;115(5):1343-1351.

4. Tanaka N, Sano K, Horiuchi A, Tanaka E, Kiyosawa K, Aoyama T. Highly purified eicosapentaenoic acid treatment improves nonalcoholic steatohepatitis. J Clin Gastroenterol. 2008;42(4):413-418.

5. Kien CL.Dietary interventions for metabolic syndrome: role of modifying dietary fats. Curr Diab Rep. 2009;9(1):43-50.

6. Wang D, Wei Y, Pagliassotti MJ. Saturated fatty acids promote endoplasmic reticulum stress and liver injury in rats with hepatic steatosis. Endocrinology.2006;147(2):943-951.

7. Van den Berg SA, Guigas B, Bijland S, et al. High levels of dietary stearate promote adiposity and deteriorate hepatic insulin sensitivity. Nutr Metab (Lond). 2010;7:24.

8. Parker HM, Johnson NA, Burdon CA, Cohn JS, O'Connor HT, George J. Omega-3 supplementation and nonalcoholic fatty liver disease: a systematic review and meta-analysis. J Hepatol. 2012;56:944-51.

9. Levy JR, Clore JN, Stevens W. Dietary n-3 polyunsaturated fatty acids decrease hepatic triglycerides in Fischer 344 rats. Hepatology 2004; 39: 608-616 [PMID: 14999679 DOI: 10.1002/hep.20093].

10. Sato, A., et al., Antiobesity Effect of Eicosapentaenoic Acid in High-Fat/High-Sucrose Diet-Induced Obesity Importance of Hepatic Lipogenesis. Diabetes, 2010. 59(10): p. 2495-2504.

11. Marsman, H.A., et al., Reversal of hepatic steatosis by omega-3 fatty acids measured non-invasively by (1) Hmagnetic resonance spectroscopy in a rat model. J Gastroenterol Hepatol, 2011. 26(2): p. 356-63.

12. Marsman, H.A., et al., Omega-3 fatty acids reduce hepatic steatosis and consequently attenuate ischemiareperfusion injury following partial hepatectomy in rats. Dig Liver Dis, 2011. 43(12): p. 984-90.

13. Duncombe, $\mathrm{W}$. The colorimeteric micro-determination of non-esterified fatty acids in plasma .clinical chimica acta $1964 ; 9(2): 122-125$.

14. Masterton GS, Plevris JN, Hayes PC. Review article: omega-3 fatty acids - a promising novel therapy for nonalcoholic fatty liver disease. Aliment Pharmacol Ther 2010; 31: 679-692 [PMID: 20415840 DOI: 10.1111/j.1365-2036.2009.04230.x]

15. Ruderman, N.B.,D. Carling, M. Pretki and J. M. Cacicedo. "AMPK, insulin resistance , and the metaboilic syndrome ". the journal of clinical investigation 2013;123 (7) :2764.

16. Bargut, T. C. L., C. A. Mandarim- de-Lacerda and M.B. Aguila. " A high fish oil diet prevents adiposity and modulates white adipose tissue inflammation pathways in mice " the journal of international biochemistery 2015;52(6):499-513. 
17. Mollace, V., M. Gliozzi, C Carresi, V. Musolino and F. Oppedisano . Re-assessing the mechanism of action of n-3 fatty acids proceedings of the Nutrition Society 2013;69 (03):324-332.

18. Nguyen MT, Satoh H, Favelyukis S, Babendure JL, Imamura T, Sbodio JI, Zalevsky J, Dahiyat BI, Chi NW, Olefsky JM. JNK and tumor necrosis factor-alpha mediate free fatty acid-induced insulin resistance in 3T3-L1 adipocytes. Journal of biological chemistery 2005;280 (42):36361-35371.

19. 19.Nicola M., Di Minno D., Russolillo A., Lupoli R., Ambrosino P., Di Minno D. Omega-3 fatty acids for the treatment of non-alcoholic fatty liver disease. World J Gastroenterol 2012 November 7; 18(41): 5839-5847.

20. Bugianesi E, McCullough AJ, Marchesini G. Insulin resistance: a metabolic pathway to chronic liver disease. Hepatology 2005;42:987-1000.

21. Andre's-Blasco.I, , Herrero-Cervera1 A, Vinue.A., Herva', PiquerasS.L., Sanz. M.,Deborah Jane Burks D.J., and Gonza'lez-Navarro. H., Hepatic lipase deficiency produces glucose intolerance, inflammation and hepatic steatosis. Journal of Endocrinology 2015; 227, 179-191.

22. Cipolla-Neto, J., Amaral, F.G., Afeche, S.C., Tan, D.X., Reiter, R.J.,. Melatonin,energy metabolism, and obesity: a review. J. Pineal Res. 2014;56, 371e381.

23. Annamalai S., Mohanam L., Prabhu AV. Effect of combination therapy of melatonin and orlistat on high fat diet induced changes in lipid profiles and liver function parameters in serum of rats.Obesity Medicine 2 (2016) 41-45.

24. Mantena S, Vaughn D, Andringa K, et al. High fat diet induces dysregulation of hepatic oxygen gradients and mitochondrial function in vivo. Biochem J 2009;417:183-93.

25. Green CJ, Hodson L. The influence of dietary fat on liver fat accumulation. Nutrients 2014;6:5018-33.

26. Shearer GC, Savinova OV, Harris WS.. Fish oil—how does it reduce plasma triglycerides? Biochim.Biophys. Acta.2012;1821:843-51

27. Horakova O, Medrikova D, van Schothorst EM, Bunschoten A, Flachs P, Kus V,Kuda O, Bardova K,Janovska K, Hensler M,Rossmeisl M, Wang-Sattler R,Prehn C, Kopeckk J. Preservation of Metabolic Flexibility in Skeletal Muscle by a Combined Use of $n-3$ PUFA and Rosiglitazone in Dietary Obese Mice. PLoS ONE 2012; 7(8):e43764.

28. Tanaka X. Zhang, E. Sugiyama, H. Kono, A. Horiuchi, T. Nakajima, H. Kanbe, E.Tanaka, F.J. Gonzalez, T. Aoyama, Eicosapentaenoic acid improves hepatic steatosis independent of PPARalpha activation through inhibition of SREBP-1 maturation in mice, Biochem. Pharmacol. 80 (2010) 1601-1612 